\title{
Article \\ Seamless Handover Scheme for MEC/SDN-Based Vehicular Networks
}

\author{
Nirmin Monir ${ }^{1}$, Maha M. Toraya ${ }^{1}$, Andrei Vladyko ${ }^{2}\left(\mathbb{D}\right.$, Ammar Muthanna ${ }^{3,4}$ (D), Mohamed A. Torad 5 , \\ Fathi E. Abd El-Samie ${ }^{6}$ and Abdelhamied A. Ateya $1,3, * \mathbb{D}$
}

check for

updates

Citation: Monir, N.; Toraya, M.M.; Vladyko, A.; Muthanna, A.; Torad, M.A.; El-Samie, F.E.A.; Ateya, A.A. Seamless Handover Scheme for MEC/SDN-Based Vehicular Networks. J. Sens. Actuator Netw. 2022, 11, 9. https://doi.org/10.3390/ jsan11010009

Academic Editors: Lei Shu, Stefan Fischer, Joel J. P. C. Rodrigues, Adnan Al-Anbuky and Mário Alves

Received: 31 December 2021

Accepted: 25 January 2022

Published: 27 January 2022

Publisher's Note: MDPI stays neutral with regard to jurisdictional claims in published maps and institutional affiliations.

Copyright: (C) 2022 by the authors. Licensee MDPI, Basel, Switzerland. This article is an open access article distributed under the terms and conditions of the Creative Commons Attribution (CC BY) license (https:// creativecommons.org/licenses/by/ $4.0 /)$.
1 Department of Electronics and Communications Engineering, Zagazig University, Zagazig 44519, Egypt; nabdelwahab@zu.edu.eg (N.M.); mahamam@zu.edu.eg (M.M.T.)

2 Faculty of Fundamental Training, The Bonch-Bruevich Saint-Petersburg State University of Telecommunications, 193232 Saint Petersburg, Russia; vladyko@sut.ru

3 Department of Applied Probability and Informatics, Peoples' Friendship University of Russia (RUDN University), 117198 Moscow, Russia; muthanna.asa@spbgut.ru

4 Department of Telecommunication Networks and Data Transmission, The Bonch-Bruevich Saint-Petersburg State University of Telecommunications, 193232 Saint Petersburg, Russia

5 Department of Communication and Electronic Engineering Higher Technological Institute, 10th of Ramadan City 44629, Egypt; Mohamed.torad@hti.edu.eg

6 Department of Electronics and Electrical Communications Engineering, Faculty of Electronic Engineering, Menoufia University, Menouf 32952,Egypt; Fathi_sayed@menofia.edu.edu

* Correspondence: a_ashraf@zu.edu.eg; Tel.: +7-9819-393-480

\begin{abstract}
With the recent advances in the fifth-generation cellular system (5G), enabling vehicular communications has become a demand. The vehicular ad hoc network (VANET) is a promising paradigm that enables the communication and interaction between vehicles and other surrounding devices, e.g., vehicle-to-vehicle (V2V) and vehicle-to-everything (V2X) communications. However, enabling such networks faces many challenges due to the mobility of vehicles. One of these challenges is the design of handover schemes that manage the communications at the intersection of coverage regions. To this end, this work considers developing a novel seamless and efficient handover scheme for V2X-based networks. The developed scheme manages the handover process while vehicles move between two neighboring roadside units (RSU). The developed mechanism is introduced for multilane bidirectional roads. The developed scheme is implemented by multiple-access edge computing (MEC) units connected to the RSUs to improve the implementation time and make the handover process faster. The considered MEC platform deploys an MEC controller that implements a control scheme of the software-defined networking (SDN) controller that manages the network. The SDN paradigm is introduced to make the handover process seamless; however, implementing such a controlling scheme by the introduction of an MEC controller achieves the process faster than going through the core network. The developed handover scheme was evaluated over the reliable platform of NS-3, and the results validated the developed scheme. The results obtained are presented and discussed.
\end{abstract}

Keywords: vehicular networks; handover; VANET; MEC; RSU; SDN

\section{Introduction}

Motivated by the high-speed development of the mobile internet and the growing business demand, the fifth-generation cellular system (5G) is supposed to have a low cost and low power consumption and be safe and reliable [1]. Fifth-generation cellular systems support new demands and requirements to enable new use cases and novel services. The transmission rate is supposedly increased up to 100 times the value of the last release of the fourth-generation cellular system $(4 \mathrm{G})$, and the peak transmission rate is expected to reach $10 \mathrm{Gbit} / \mathrm{s}[2,3]$. One of the main targets for $5 \mathrm{G}$ is to reduce the end-to-end delay to $10 \mathrm{~ms}$ for the first commercial releases and to $1 \mathrm{~ms}$ ( $5 \mathrm{~ms}$ while moving) to support ultrareliable 
low-latency use cases such as vehicular communications, augmented and virtual reality (AR, VR), and the Tactile Internet [4,5]. Furthermore, 5G is expected to achieve a spectrum efficiency improvement of 5 to 10 times, and the user experience can be guaranteed at a speed of $500 \mathrm{~km} / \mathrm{h}$. Fifth-generation cellular systems face many challenges, including the massive traffic that is expected to be 1000 times that of the existing systems $[6,7]$.

One of the main objectives of $5 \mathrm{G}$ systems is to improve the end-user experience by providing current services and introducing new services and applications with low/ultralow latency, a high data rate, ultrahigh reliability, and ultrahigh availability [8]. Vehicular communication is one of these $5 \mathrm{G}$ use cases, which is intended to provide the infrastructure and coverage for vehicles with heterogenous mobility [9].

Vehicular systems are inseparable components of a smart city environment due to several applications that improve the quality of life, safety, and security of the city [10]. Vehicular networks provide the infrastructure and communication coverage to vehicular devices with heterogenous mobilities, including high mobility. The applications of vehicular networks vary from safety applications such as blind-spot warning and traffic light violations to entertainment such as streaming media and convenience such as parking space identification [11,12]. Recently, many standards have been established for vehicular communications, such as the IEEE 802.11p and IEEE 1609 family group [13,14]. These standards achieve effective communication between vehicles and infrastructure. However, vehicular ad hoc networks (VANETs) are still considered a challenging form of the wireless communication technology that complements intelligent transportation systems (ITS) aiming to improve transportation in cooperation with information and communication technologies (ICTs) [15]. VANETs are mobile ad hoc networks (MANETs); however, protocols that perform well in MANETs may not be a great fit for VANETs, due to the usage conditions of high mobility, intermittent connectivity, and heterogeneity.

The dramatic increase in the number of vehicles, especially in crowded cities, adds more constraints to the network design. High traffic density introduces data traffic overload, particularly when using broadband access services such as transferring a video from a vehicle or a roadside unit (RSU) to solve a transport management problem [16,17]. Thus, new technologies, such as mobile edge computing (MEC), software-defined networking, SDN, and blockchain, should be used to provide the required network scalability, reliability, and availability [18].

One of the main challenges with VANETs is the handover process [19]. Handover, or handoff, is the process in mobile communication and telecommunication systems in which a connected cellular call or data session is transferred from one base station or cell site to another, without the session becoming disconnected. Cellular services are based on mobility management; thus, handover allows users to move from one cell site range to another or switch to the nearest cell site for better performance [20]. Handoff can also be described as a process in which a link is transferred from one base station to another base station due to a lack of signal strength. Several operations are included in the handoff process, such as initiating the handoff, allocating the channel, breaking the connection with the old base station, and choosing the new base station [21].

In VANETs, due to high mobility, users will move from the coverage of one base station, i.e., an RSU with an eNB, to another, and thus a handover mechanism should be deployed efficiently and in a seamless way [22,23]. This work provides a novel handover mechanism to perform, manage, and control the handover process of a VANET that runs over a $5 \mathrm{G}$ cellular system.

Mobile edge computing (MEC) is a recent communication technology that cellular network operators have introduced to improve network efficiency [24]. This can be achieved by offloading computing workloads to nearby clouds instead of going through the core network to the remote cloud [25].

Deploying MEC for communication systems achieves various benefits, including higher system reliability, availability, and spectrum efficiency [26]. Furthermore, MEC 
enables the network operator to introduce new services and provide a new means of service innovation [27].

In this work, we use MEC as part of a vehicular network in which cars can communicate with smart traffic lights, surrounding objects, other vehicles on the road, and other infrastructure. The main contributions of this article can be summarized in the following points:

1. Designing and developing a reliable system structure for a VANET over a 5G system, with the deployment of MEC technology, that supports V2X and V2V communications.

2. Designing and developing a novel seamless, fast handover mechanism for the introduced VANET system structure.

3. Implementing the developed handover algorithm over an MEC server.

4. Conducting a performance evaluation of the proposed system and the developed handover mechanism.

\section{Background and Related Works}

Recently, the VANET has attracted many researchers in the academic and industrial fields $[28,29]$. The VANET is one of the main use cases of $5 \mathrm{G}$ networks that has been announced, and since the first commercial version of the 5G system was released, the research in the area of VANETs has become more attractive [30,31]. Many existing works in the literature consider VANETs; however, in this section, we consider only the works that are most relevant to our proposed study in order to highlight the novelty of our work.

The handover process is one of the main critical processes in vehicular communication systems, since it should be done in an efficient, seamless way [32]. This is crucial and presents a challenge for designing VANETs, due to the high mobility of vehicles $[33,34]$. There are few works that consider the handover process in VANETs or the development of a handover algorithm for VANETs. We considered the recently developed handover schemes for VANETs.

In [35], the authors introduced a novel network structure for a VANET based on MEC technology. The proposed structure achieved high system scalability and high latency efficiency. However, the handover mechanism considered was that of a traditional cellular system, essentially the LTE-V handover mechanism. The authors did not consider developing a new handover mechanism for the developed VANET structure. This would affect the overall performance and create conflict, since MEC servers were not considered in the handover process. In our proposed work, the MEC paradigm is deployed in the VANET structure and represents a main part of the suggested handover algorithm. This makes the handover process more seamless and achieves many other benefits, as introduced later, in Section 3.

In [36], the authors developed a novel network structure for vehicular communications based on SDN technology. The developed system met the ITS requirements and the specifications that had been announced. The challenge of the handover scheme was addressed by developing a fog-shaped cell that reduced the rate of the handover process among RSUs. The work considered the throughput and latency as the key performance indicators. The developed system achieved a higher latency and throughput than other existing systems.

In [37], the authors developed an SDN-based handover scheme for vehicular networks. SDN was used to maintain the transport layer communication during the handover process. The VANET deployed the IEEE 802.11p and enabled V2X communications over an unlicensed spectrum. The simulation results validated the system in terms of throughput. However, our proposed system involves implementing the handover scheme over MEC servers, which achieves less latency. Moreover, the time required to migrate V2X tasks is considered, which reduces the probability of task blocking during the handover process and achieves a higher system availability.

In [38], scientists employed mobile edge computing (MEC) and software-defined network (SDN) technologies in the building of the network to modify the overall net- 
work performance under the conditions of high traffic density. They also developed an algorithm of D2D-based clustering to solve the problem of disconnected vehicles in dead zones and offload the network in regions with high traffic. By using the aforementioned technologies, the authors could achieve a performance of up to $74 \%$ gains in terms of task-blocking probability.

In [22], the authors proposed an advanced handover algorithm by adopting SDN in the building of the network architecture, and they explained the advantages of using SDN in the network control to administer the data transfer in VANETs. Hence, the connection of the transmission layers could be fixed when the handover occurred. This achieved a high-quality improvement based on the vehicle's mobility and link rates.

In [39], the authors focused their studies on the latest developments in data-offloading techniques in VANETs. Specifically, they investigated the communication models between vehicles and infrastructure and classified the technique into several categories: data offloading through vehicle-to-vehicle communication, data offloading through vehicle-to-everything communication, and data offloading through vehicle-toinfrastructure communications.

\section{MEC/SDN Vehicular Network Structure}

In this section, the considered structure of the VANET is introduced. Figure 1 presents the developed MEC/SDN vehicular network that runs over the 5G system. The system enables V2V and V2X communications, as vehicles can communicate with each other and with distributed roadside units (RSUs). Each vehicle deploys an onboard unit (OBU) whose structure is presented in Figure 2. This embedded unit facilitates the implementation and execution of the required communication standards and interfaces.

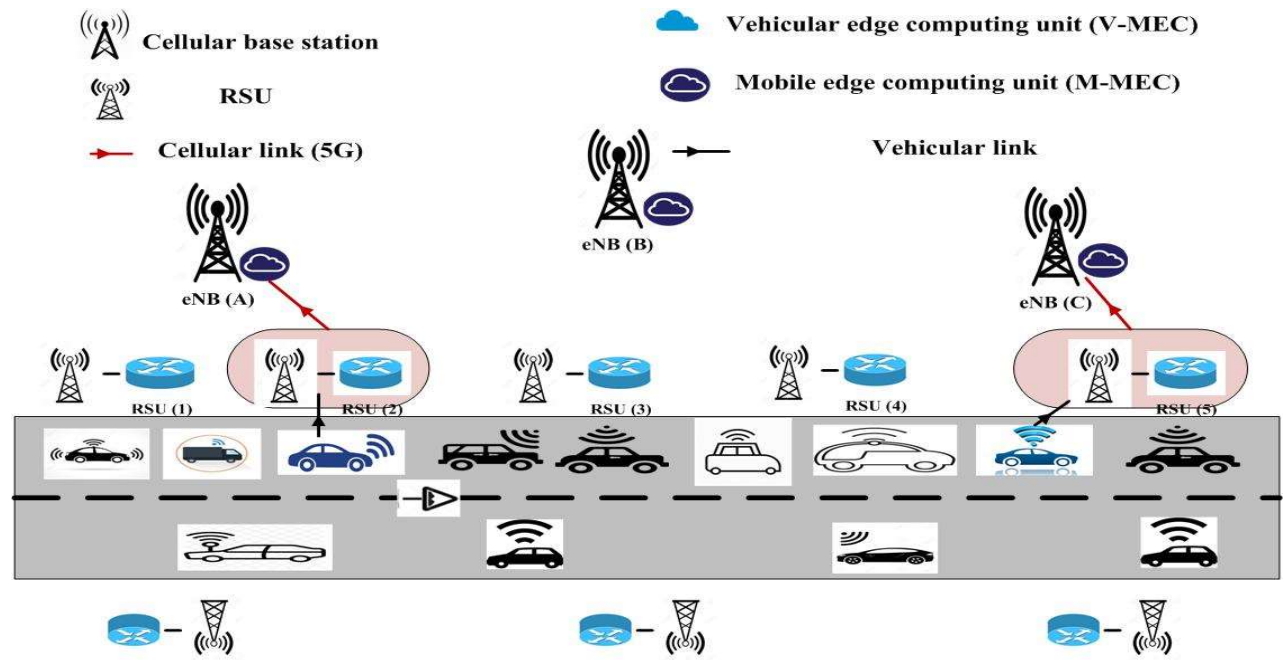

Figure 1. System structure of the developed MEC-based vehicular network. 


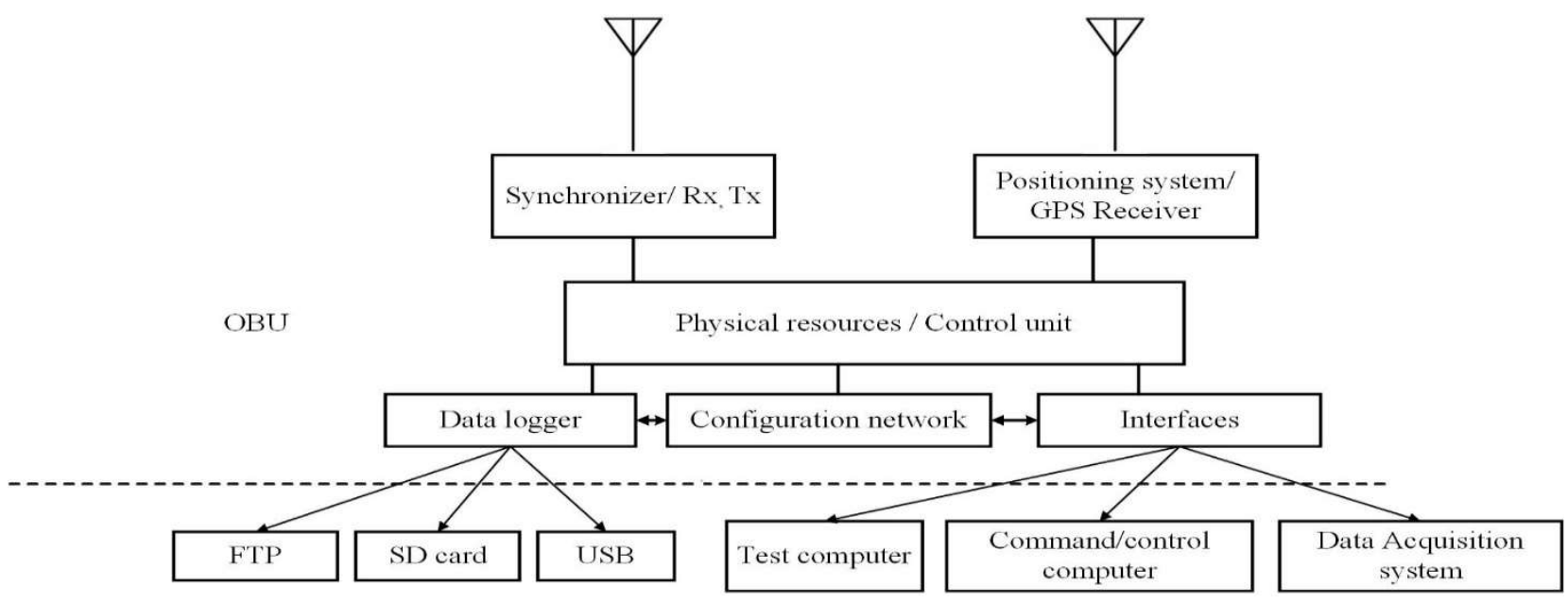

Figure 2. Structure of the OBU.

The developed structure deploys distributed edge computing with two heterogeneous levels. The first level represents the multiple-access edge computing units connected to the RSUs, which are referred to as the vehicular edge computing units (V-MECs). The second level represents the distributed MEC units connected to the cellular base stations, which are referred to as the cellular MECs (M-MECs). Both levels of these distributed edge units are introduced to provide computing resources near to vehicles to achieve higher latency efficiency, higher reliability, and higher system availability. Moreover, the introduction of MEC units facilitates the implementation of the developed networking algorithms. In particular, the V-MEC implements our developed handover scheme, resulting in a higher execution efficiency than that of the traditional systems.

The introduction of distributed edge units as presented in Figure 1 facilitates the achievement of the required quality of service (QoS) of V2X applications. The communication between the OBU and the V-MEC reduces the communication latency, increases the throughput, achieves a higher availability and reliability, and increases the spectral efficiency.

The network deploys SDN technology for the control and management scheme. The considered core network deploys a control plane with multiple SDN controllers. The dataplane of the SDN deploys a distributed scheme of OpenFlow switches that receive their forwarding tables and their updates from the control scheme over the OpenFlow interface. The introduction of SDN technology is intended to provide the required network flexibility and an efficient management scheme for the network. For the developed handover scheme, the introduction of SDN achieves a seamless process, since the TCP connection is maintained.

\section{Novel Seamless Handover Scheme for MEC/SDN Vehicular Networks}

When a vehicle comes to the intersection between two coverage regions of neighboring RSUs, the vehicle should perform a seamless, fast handover process. Setting up the handover process and selecting the optimum time to turn from one coverage region of an RSU to the other coverage region is a challenge, due to vehicles' mobility. This section presents the developed seamless, fast handover scheme for the considered vehicular network structure introduced in the previous section.

The quality of the communication process depends on the received signal power, i.e., the received signal-to-interference noise ratio $\left(\mathrm{SINR}_{\mathrm{Rx}}\right)$, which should not be decreased lower than a threshold level. The main steps of the developed handover scheme are presented in Figure 3; Figure 4 shows the signaling flow of the developed handover scheme. The first step is the initialization phase, when the vehicle triggers the handover according to the gathered information. These data contain parameters gathered by the 
different communication layers, e.g., the transport and network layers, such as the signaling parameters, the spectrum parameters, the mobility, and the throughput. Once the received signal strength decreases below an initial threshold level $\left(\mathrm{SINR}_{\mathrm{Rx} \text {-th-1 }}\right)$, the vehicle turns from the steady state to the inspection state. In the inspection state, the vehicle calculates the expected time to reach the next threshold level of received power $\left(\mathrm{SINR}_{\mathrm{Rx}-\mathrm{th}-2}\right)$, at which the handover process should start.

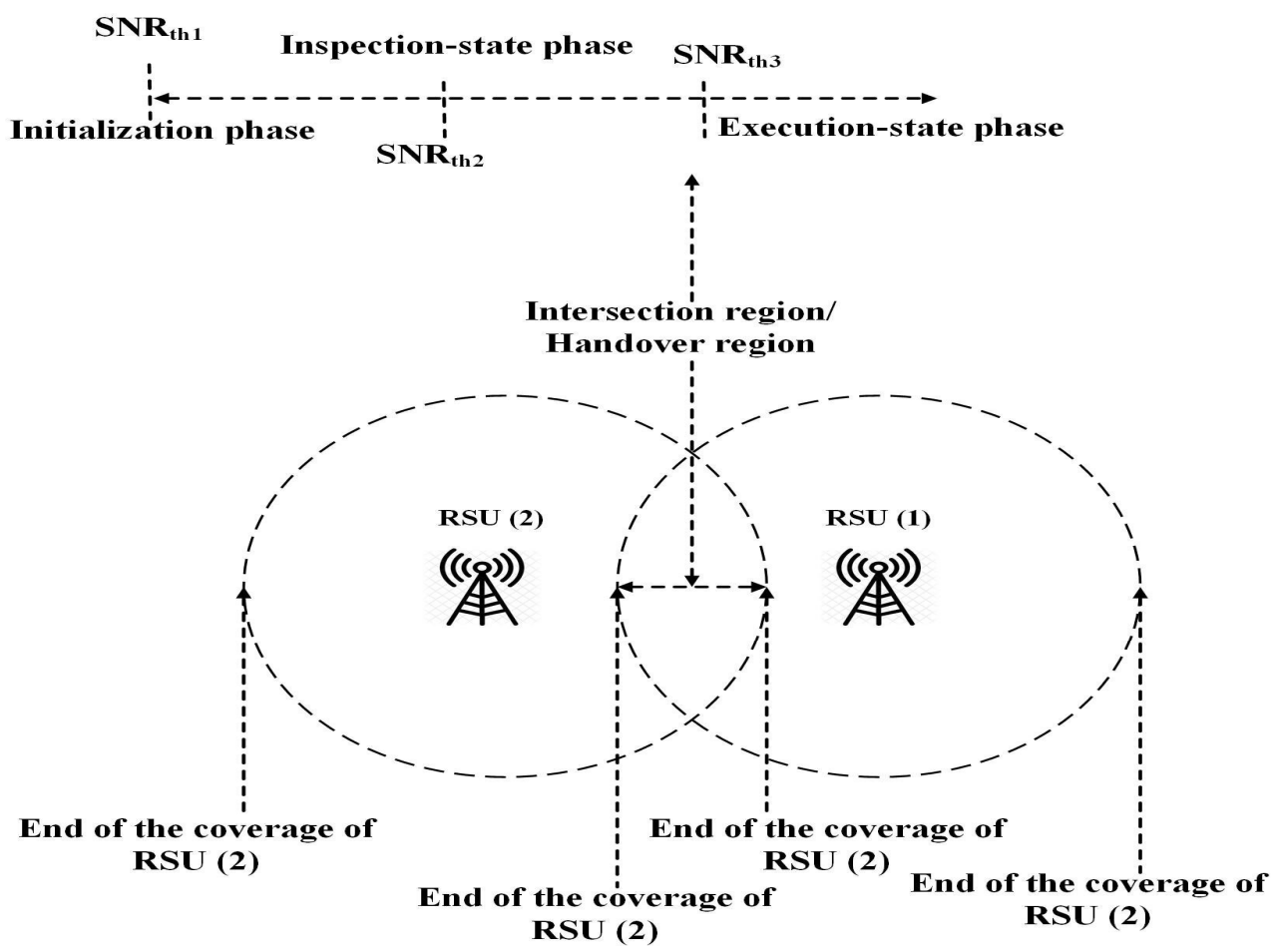

Figure 3. Handover stages at intersection of coverage regions.

In the inspection state, the OBU communicates the MEC server of the serving base station to set up a connection with the MEC server of the targeted RSU. The serving MEC server receives information on the vehicle's current mobility and position. The MEC server calculates the expected time to perform the handover process according to the measured data. It then sends a handover request to the targeted MEC server with the expected time. The targeted MEC server starts to set up a connection with the relevant vehicle through the targeted base station at the estimated time. When reaching the threshold level SINR Rx-th-2, $_{\text {, }}$ the connection with the targeted MEC server should be set up, and the third phase of the handover mechanism, i.e., the execution state, starts.

In the execution state, the handover process should be performed, and the vehicle should establish a connection to the targeted coverage region, while the connection to the currently serving coverage region should be disabled. This process should be carried out seamlessly for the end-user, with no discontinuity of the communication process. This requires migrating the current tasks of the vehicle from the current MEC server to the targeted MEC server. This takes a length of time that should be calculated in the second phase, i.e., the inspection state, as the threshold power SINR $\mathrm{Rx}_{\text {-th-2 }}$ is allocated according to this length of time. During the migration process, the vehicle starts sending information to the targeted RSU. After the process ends, the connection with the old RSU is ended through the MEC server. This should be done before the received power reaches the third threshold level (SINR $\mathrm{Rx}_{\mathrm{th}-3}$ ), which represents the critical received signal, after which the packet reception will be affected.

To perform a seamless handover process, we introduce SDN. The SDN controller maintains the connection at the transport layer unaltered, thus avoiding the limitations of the congestion window (cwnd). To perform a faster migration process and am efficient 
handover process, we introduce the facilities of SDN to the distributed edge computing servers. This can be achieved by our previously developed MEC platform, which deploys an MEC controller with an interface to the SDN controller and implements a lightweight version of the control scheme.

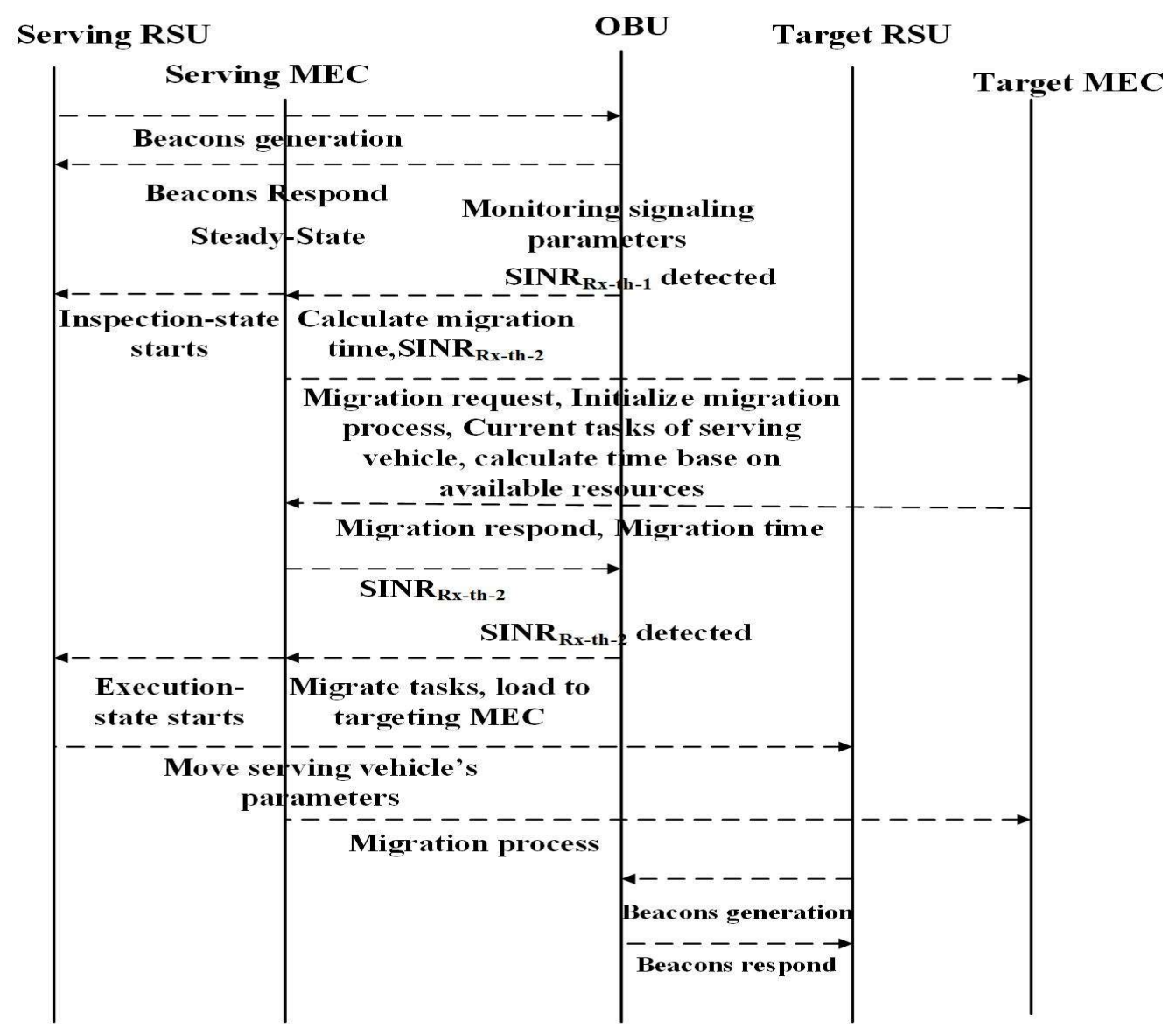

Figure 4. Signaling flow of the developed handover scheme.

\subsection{Estimating Threshold Levels}

Figure 4 presents the three considered threshold levels deployed for the developed fast handover mechanism. The first threshold level, $\mathrm{L}_{1}$, represents the starting point of the developed handover mechanism, i.e., the start of the inspection state. The second threshold level, $\mathrm{L}_{2}$, is variable, and it represents the end of the inspection state and the start of the execution phase of the handover process. The handover process must be ended before the third level, $\mathrm{L}_{3}$, at which the packet reception ratio from the current serving base station decreases below the required level. Thus, the handover process should be ended before this point to maintain communication with the required QoS.

The main factor in deciding the handover process is the value of the received signal that results in an acceptable packet reception ratio (PRR). Once the value of the received packet ratio decreases below the critical PRR, the communication is interrupted and the quality of service (QoS) is decreased below the required level. Thus, the handover process must be performed before the received packet ratio decreases below the critical PRR. The probability of successful packet reception, $\mathrm{P}_{\mathrm{r}, \mathrm{sr}}$, is calculated by Equation (1).

$$
\begin{gathered}
P_{r, s r}=P\left(L(d B) \leq P_{T x}(d B)-P_{R x}(d B)\right)=P\left(S_{\left.N N R_{R x} \geq S I N R_{R x-t h-3}\right),},\right. \\
L(d B)=n_{0} B_{\text {Wave }}(d B)+P L(d)(d B),
\end{gathered}
$$


where $\mathrm{L}$ is the communication losses, $\mathrm{P}_{\mathrm{Tx}}$ and $\mathrm{P}_{\mathrm{Rx}}$ are the total transmitted and received power, SINR $_{R x}$ is the signal-to-noise ratio of the received signal, and SINR $_{R x-t h-3}$ is the threshold of the received signal-to-noise ratio after which the packets cannot be received successfully. The communication losses can be calculated as the sum of the channel loss and the path loss (PL), which can be calculated as a function of the communication distance (d) using the street canyon model as introduced in [40]. The power spectral density of the considered $60 \mathrm{GHz}$ spectrum is $\mathrm{n}_{0}$, and the Bwave is the allocated bandwidth of the wireless access of the vehicular environment (wave). Thus, to guarantee the successful reception of packets, the received signal-to-noise ratio must be higher than the threshold level, SINR $\mathrm{Rx}_{\mathrm{th}-3}$. This condition is included in the developed handover mechanism, as the handover process should be performed before SINR $_{R x}$ decreases below the threshold level of SINR Rx-th-3.

During the inspection state, the initial MEC server calculates the time required to migrate the current services of the vehicle, $\mathrm{T}_{\mathrm{m}}$. Based on the received data about the vehicle's mobility, $\mathrm{V}_{\mathrm{i}}$, and the vehicle's current position, the MEC server calculates the distance corresponding to the $\mathrm{T}_{\mathrm{m}}$. This is the required distance a vehicle should travel during the migration process and is referred to as $\mathrm{dm}$. If the distance between the first threshold level, $\mathrm{L}_{1}$, to the third threshold level, $\mathrm{L}_{3}$, is $\mathrm{d}$, the threshold level $\mathrm{L}_{2}$ is chosen to achieve $d_{2}>d_{m}$.

\subsection{Calculating Packet Error Rate}

One of the main performance indicators is the packet error rate (PER), which is an indicator for failed transmission. The PER represents the ratio of undelivered packets to the total transmitted packets. It is measured during a sliding window. Figure 5 indicates the methodology of evaluating the PER. The total interval during which the PER is calculated is divided into $\mathrm{k}$ windows $(\omega)$ of equal time intervals $(\tau)$. The total PER of a vehicle $v_{i}$, $\mathrm{PER}_{\mathrm{T}}{ }^{\mathrm{vi}}$, is calculated by Equation (3).

$$
P E R_{T}^{V i}=\sum_{j=1}^{k} P E R_{\omega j}^{v i}
$$

where $\mathrm{PER}_{\omega j}{ }_{\mathrm{j}}^{\mathrm{vi}}$ is the PER of the jth window and can be calculated as the ratio of undelivered packets to the total transmitted packets during the window. PER is an important performance metric, since it indicates two main packet losses; the loss of packets missed during queuing due to the arrival of a newer basic safety message (BSM) and the packet loss in the channel due to collision or weak signal.

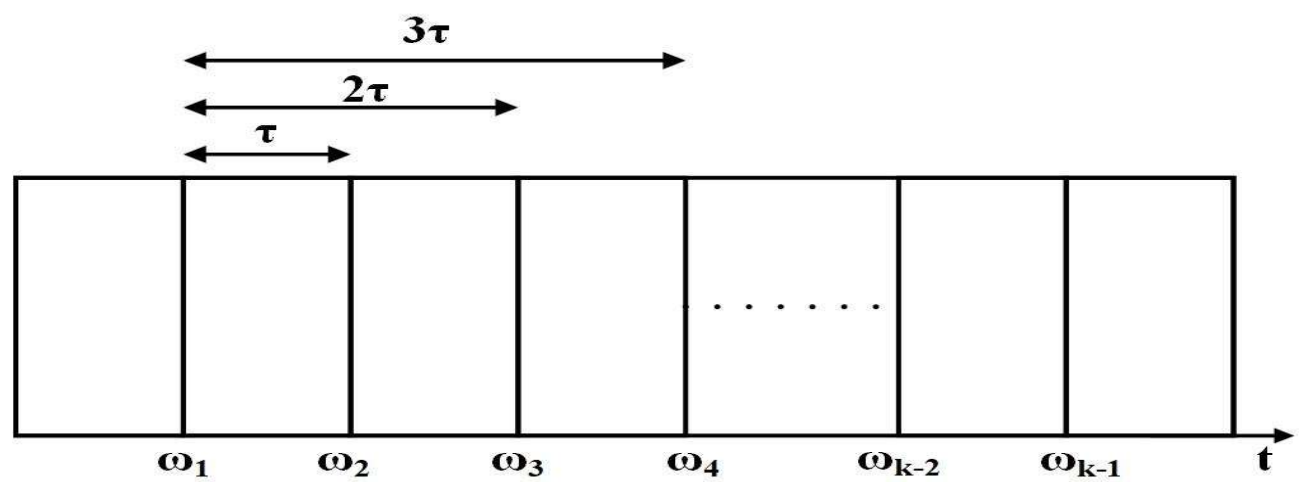

Figure 5. Sliding windows to calculate PER.

\section{Performance Evaluation}

The developed handover scheme for an MEC/SDN VANET is evaluated in this section. Various simulation scenarios are considered, and the results are presented and discussed. 


\subsection{Simulation Setup}

The system was evaluated over the NS-3 platform, with an open-source traffic simulation from the Simulation of Urban Mobility (SUMO) to provide vehicle mobility. The considered vehicles' mobility was that of the highway. The simulation process ran over a machine with an Intel Core i9 processor and 64GB RAM. All the required SDN modules for the SDN controller and OpenFlow switches were included with OpenFlow version 1.3.

The considered simulation topology consisted of the specifications presented in Table 1. A two-lane bidirectional road of $5 \mathrm{Km}$ length was considered for the simulation process. The road was served by five RSU units, with one MEC server connected to each RSU. The specifications of the RSUs and the connected MEC servers are presented in Table 1.

Table 1. Simulation parameters.

\begin{tabular}{|c|c|}
\hline Parameter & Value \\
\hline Road length & $5 \mathrm{Km}$ \\
\hline Number of vehicles $(\mathrm{N})$ & $N \in\{200,400,600\}$ \\
\hline Number of RSUs & 5 \\
\hline Number of MEC units & 5 \\
\hline MEC unit placement & Equidistant \\
\hline Vehicle velocity & $\mathrm{V}_{\mathrm{i}} \in\{30,40,50,60,70\} \mathrm{Km} / \mathrm{h}$ \\
\hline Traffic density & $\mathrm{Td}_{\mathrm{vi}} \in\{0.1,0.2,0.3\}$ veh. $/ \mathrm{m}$ \\
\hline Radio transmission range of RSUs & $500 \mathrm{~m}$ \\
\hline Maximum transmission level & $20 \mathrm{dBm}$ \\
\hline Storage/RAM (MEC) & $2048 \mathrm{Mb}$ \\
\hline Storage/HDD (MEC) & $5 \mathrm{~Gb}$ \\
\hline Processing/CPU (MEC) & $\epsilon\{0.7,2.5\} \mathrm{GHz}$ \\
\hline Maximum workload & 50 events/s \\
\hline Service rate & $10 \mathrm{Mbps}$ \\
\hline Streaming service bandwidth & $\epsilon\{10,2048\}(\mathrm{Kb} / \mathrm{S})$ \\
\hline Vehicle task energy & $\epsilon\{20,80\}$ (watt/s) \\
\hline Initial threshold level, $\mathrm{SNR}_{\mathrm{Rx} \text {-th-1 }}$ & $30 \mathrm{~dB}$ \\
\hline Third threshold level, $\mathrm{SNR}_{\mathrm{Rx} \text {-th-3 }}$ & $20 \mathrm{~dB}$ \\
\hline Data rate & $18 \mathrm{Mbps}$ \\
\hline Channel bandwidth & $10 \mathrm{MHz}$ \\
\hline Receiver sensitivity & $-94.0 \mathrm{dBm}$ \\
\hline Packet transmission frequency & $10 \mathrm{~Hz}$ \\
\hline Packet size & 190 bytes \\
\hline OpenFlow switch & 1.3 \\
\hline Service rate of controller & $30,000 \mathrm{req} / \mathrm{s}$ \\
\hline Average request rate of switch & $\epsilon\{1500,3000\}$ \\
\hline SDN controller processing delay & $0.5 \mu \mathrm{s}$ \\
\hline OpenFlow switch processing delay & $5 \mu \mathrm{s}$ \\
\hline
\end{tabular}

The vehicles were distributed along the road according to the traffic density shown in Table 1. Three values for the total number of deployed vehicles, $\mathrm{N}$, were considered to investigate the effect of the increase in traffic along the road on the performance of the developed handover scheme. Moreover, three different traffic densities were considered to investigate the effect of the change in the traffic density on the overall network performance. We considered our previously developed offloading scheme introduced in [41] for data offloading in the proposed MEC-based VANET. The offloading parameters are given in Table 1.

Each vehicle in the developed system was assigned a computing task corresponding to the real task's workload. For the performance evaluation, we considered three performance metrics: the packet delivery ratio (PDR), the percentage of blocked computing tasks, and the percentage of failed handover processes from the total number of required handover processes. 


\subsection{Simulation Results}

Figures 6 and 7 present the recorded PDR for the developed MEC-based handover scheme and the traditional handover scheme without the MEC involvement. Figure 6 provides the PDR for both systems at different traffic densities, with the distance to the RSU. These results were for a VNET with 400 vehicles, i.e., $N=400$, and the vehicles' speed was $50 \mathrm{Km} / \mathrm{h}$. Up to $300 \mathrm{~m}$ away from the RSU, both handover schemes achieved almost the same PDR; however, after $300 \mathrm{~m}$, the value of the PDR for both systems changed. This change increased after $400 \mathrm{~m}$, since at this distance the handover process had started or was close to starting in the traditional handover scheme. Moreover, with the increase in the traffic density, the PDR decreased due to the higher probability of collisions.

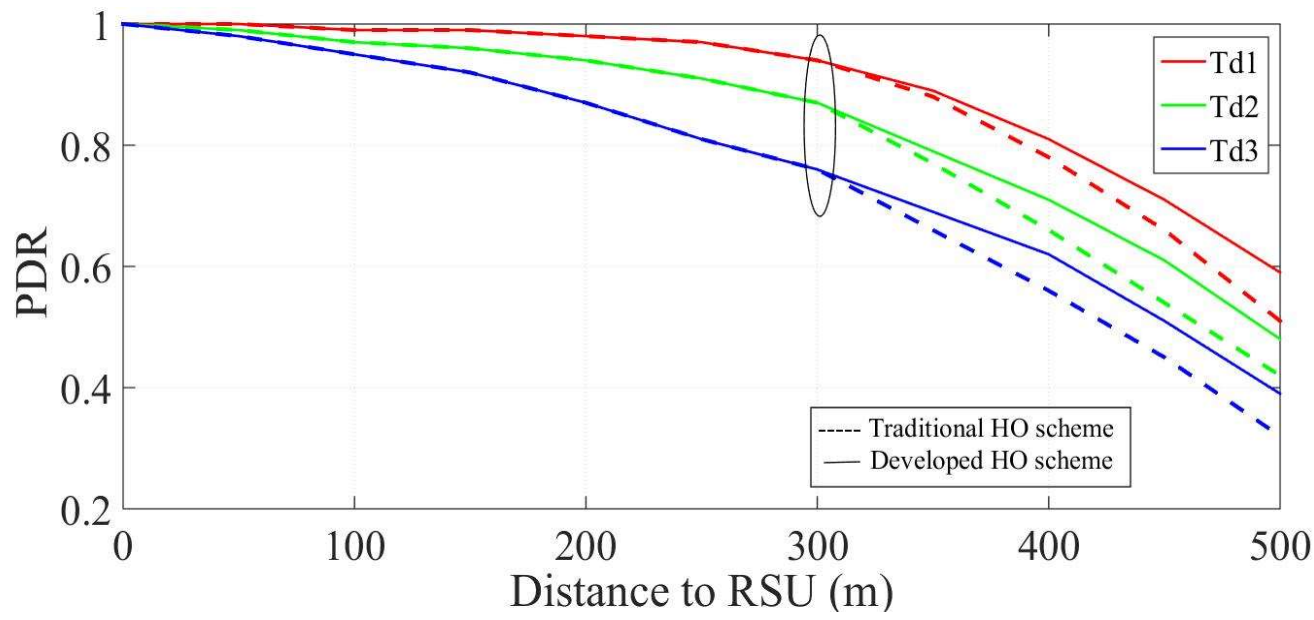

Figure 6. PDR at different traffic densities, with the distance to RSU.

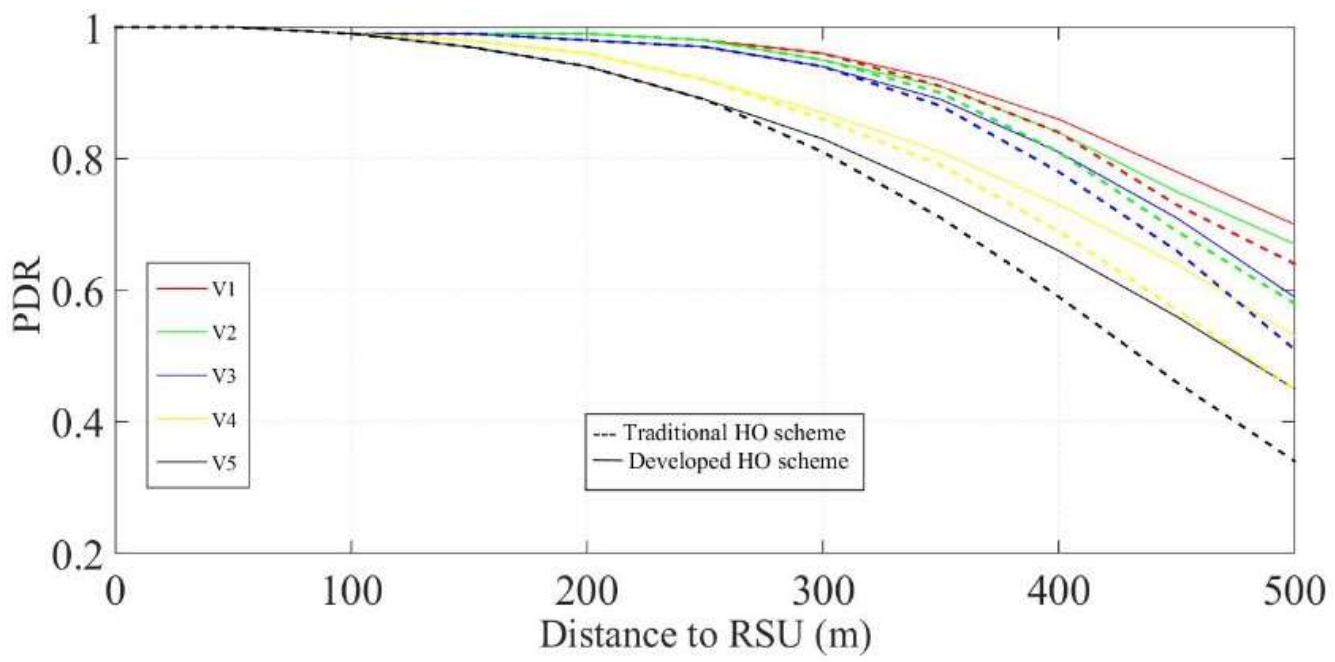

Figure 7. PDR at different vehicle velocities, with the distance to RSU.

Figure 7 provides the PDR for both systems, the proposed and the traditional handover scheme, at different vehicle velocities. These results were for a VNET with 400 vehicles, i.e., $\mathrm{N}=400$, and a traffic density of $0.1 \mathrm{veh} / \mathrm{m}$. Five vehicle velocities were considered, i.e., those given in Table 1. With the increase in the vehicle velocity, the PDR decreased. Up to $50 \mathrm{Km} / \mathrm{h}$ the change in the PDR for both systems due to different velocities was small; however, it increased for the 60 and $70 \mathrm{~km} / \mathrm{h}$ velocities. The developed MEC/SDNbased handover scheme achieved an improvement in the PDR compared to the traditional handover scheme by an average of $47 \%$. Furthermore, the developed scheme achieved a greater improvement in the PDR for a high traffic density and high vehicle velocity 
compared to the traditional scheme. The percentage improvement in the PDR in the case of high vehicle velocity was $64 \%$.

The second performance metric considered was the percentage of blocked tasks. Three systems were considered for the performance evaluation: the first system represented the traditional VANET with the traditional handover scheme; the second system represented the MEC-based VANET with a traditional handover scheme; and the third system represented the developed MEC/SDN-based VANET with the developed handover scheme. For the three systems, our previously developed offloading scheme, introduced in [41], was used. Figures 8-10 provide the three systems' percentage of blocked tasks in different scenarios. The statistical analysis of the results for each of the three systems is presented in Table 2. This includes the mean and the standard deviation (Std) of the results for each system.

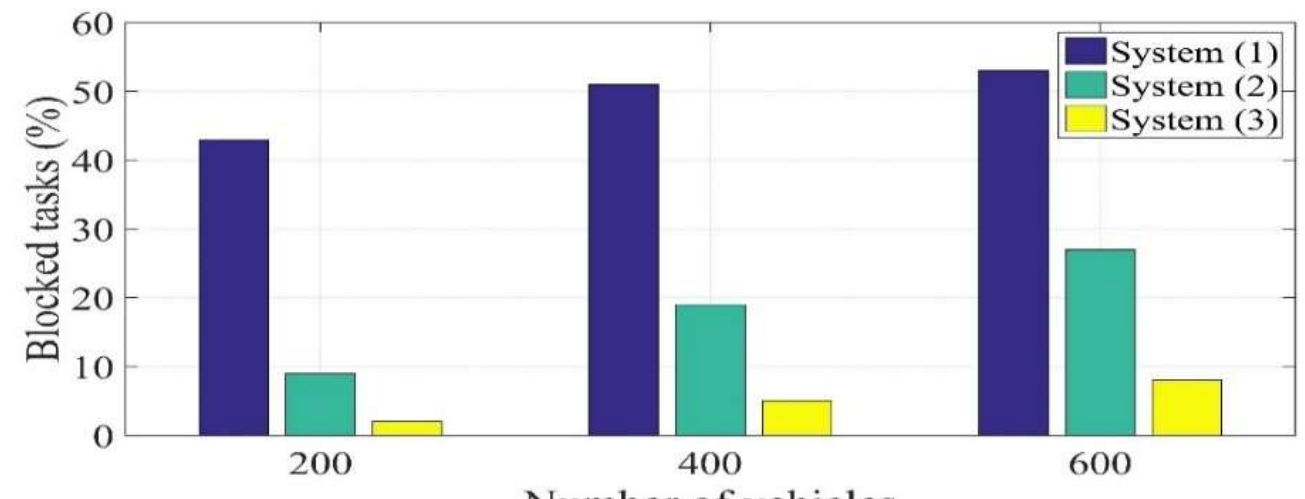

Figure 8. Percentage of blocked tasks for the three systems with different numbers of vehicles.

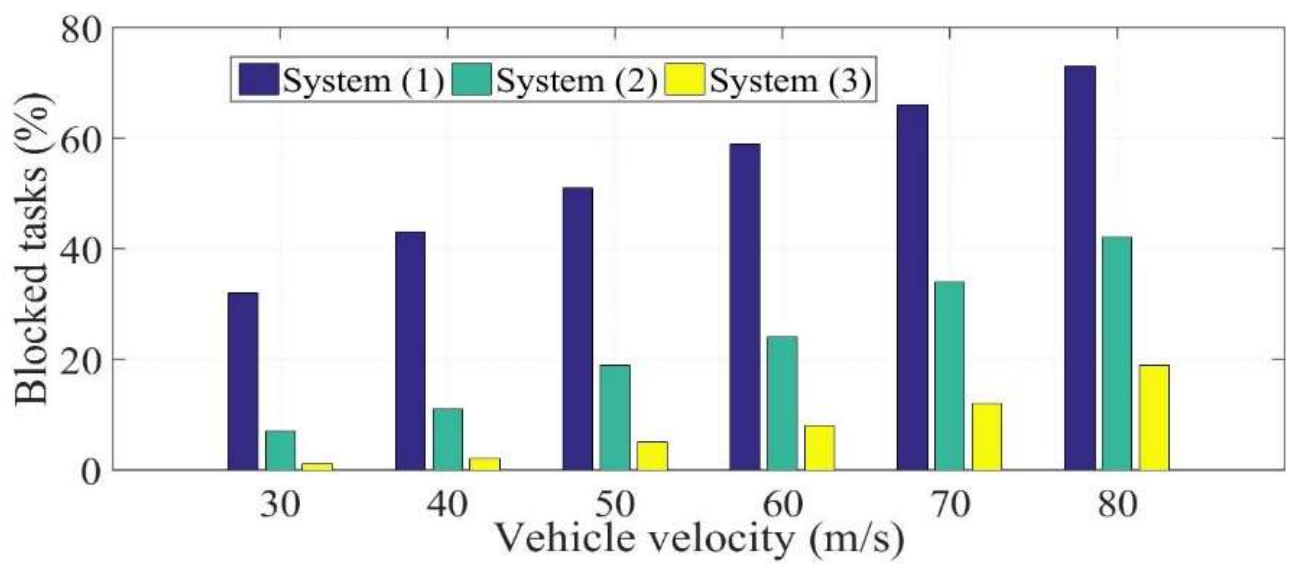

Figure 9. Percentage of blocked tasks for the three systems at different vehicle velocities. 


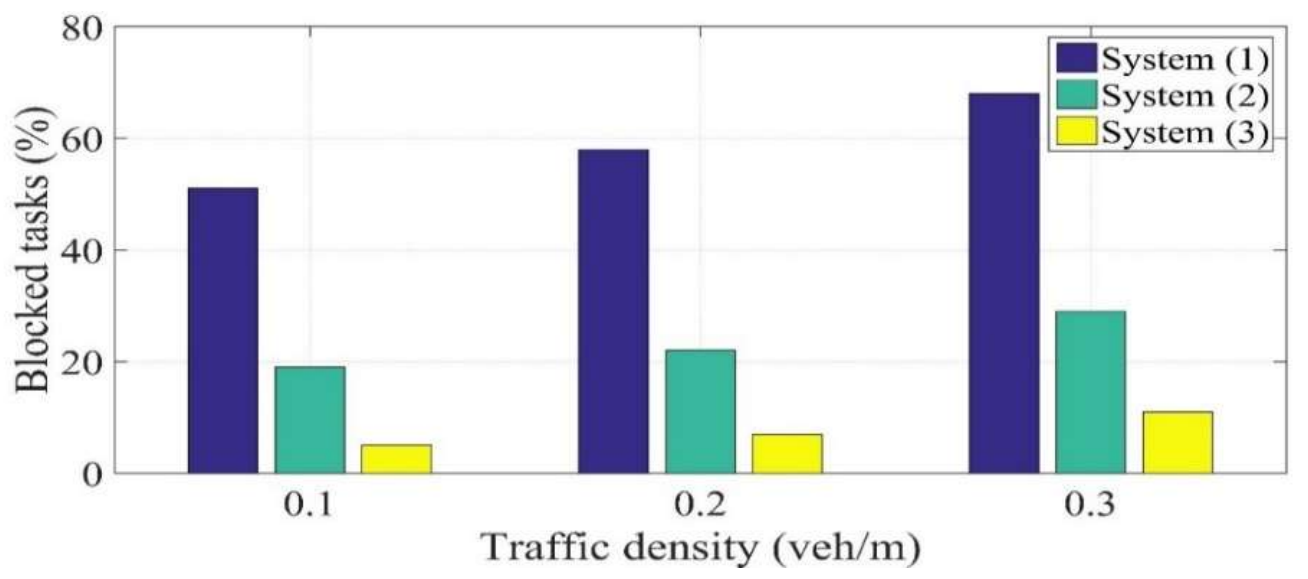

Figure 10. Percentage of blocked tasks for the three systems at different traffic densities.

Table 2. Statistical analysis of simulation results of the three systems introduced in Figures 8-10.

\begin{tabular}{ccccccc}
\hline \multirow{2}{*}{ Figure } & \multicolumn{2}{c}{ System (1) } & \multicolumn{2}{c}{ System (2) } & \multicolumn{2}{c}{ System (3) } \\
\cline { 2 - 7 } & Standard Deviation & Mean & Standard Deviation & Mean & Standard Deviation & Mean \\
\hline Figure 8 & 5.29 & 49.00 & 9.02 & 18.33 & 3.00 & 5.00 \\
Figure 9 & 15.13 & 54.00 & 5.41 & 22.83 & 6.79 & 7.83 \\
Figure 10 & 8.54 & 59.00 & 5.13 & 23.33 & 3.06 & 7.67 \\
\hline
\end{tabular}

Figure 8 provides the average percentage of blocked tasks for each system in three scenarios with different numbers of deployed vehicles. The developed MEC/SDN-based system achieved a better performance in terms of blocked tasks compared with the other two system. This performance improvement increased with the increase in the number of vehicles in the network. This was due to the introduction of the MEC units managed by SDN, which provided a path for data offloading. Moreover, the developed handover scheme reduced the probability of task blocking because of the consideration of the migration time of the computing tasks.

Figure 9 provides the percentage of blocked tasks for the three systems at different vehicle velocities. These results were for a VANET with 400 vehicles, i.e., $\mathrm{N}=400$, and a traffic density of $0.1 \mathrm{veh} / \mathrm{m}$. The percentage of blocked tasks increased in all three systems with the increase in the vehicle's velocity. However, the percentage increase in the number of blocked tasks for the developed scheme was the lowest. Figure 10 shows the percentage of blocked tasks for the three systems at different values of traffic density. The three systems were simulated for 400 vehicles, i.e., $\mathrm{N}=400$, and a vehicle velocity of $50 \mathrm{Km} / \mathrm{h}$. The percentage of blocked tasks increased with the increase in traffic density in all three systems; however, the developed scheme achieved a higher efficiency in terms of blocked tasks. The developed MEC/SDN-based handover scheme achieved an average percentage improvement in the number of blocked tasks of $51 \%$ compared to the first system. Thus, the introduction of MEC in the handover scheme achieved a higher efficiency in terms of blocked tasks, due to the implementation of the handover scheme near to the end user, with less overhead. Moreover, the deployment of SDN with MEC facilitated the operation of the MEC during the handover implementation and the maintenance of the TCP connection.

The third performance metric was the percentage of failed handover processes compared to the total number of requested handover processes. Figures 11-13 provide the records of the handover failure rate for the developed handover scheme and the traditional schemes. These results were for different velocities, traffic densities, and numbers of deployed vehicles. Figure 11 provides the handover failure rate for the two systems at different traffic densities. These results were for a VANET with 400 vehicles and a vehicle velocity of $50 \mathrm{Km} / \mathrm{h}$. The developed scheme achieved better efficiency in performing the 
handover process. Figure 12 provides the handover failure rate at different velocities, while Figure 13 provides the failure rate at different values of the number of deployed vehicles. The developed handover scheme achieved an average percentage improvement in the failure rate of the handover process of $18 \%$.

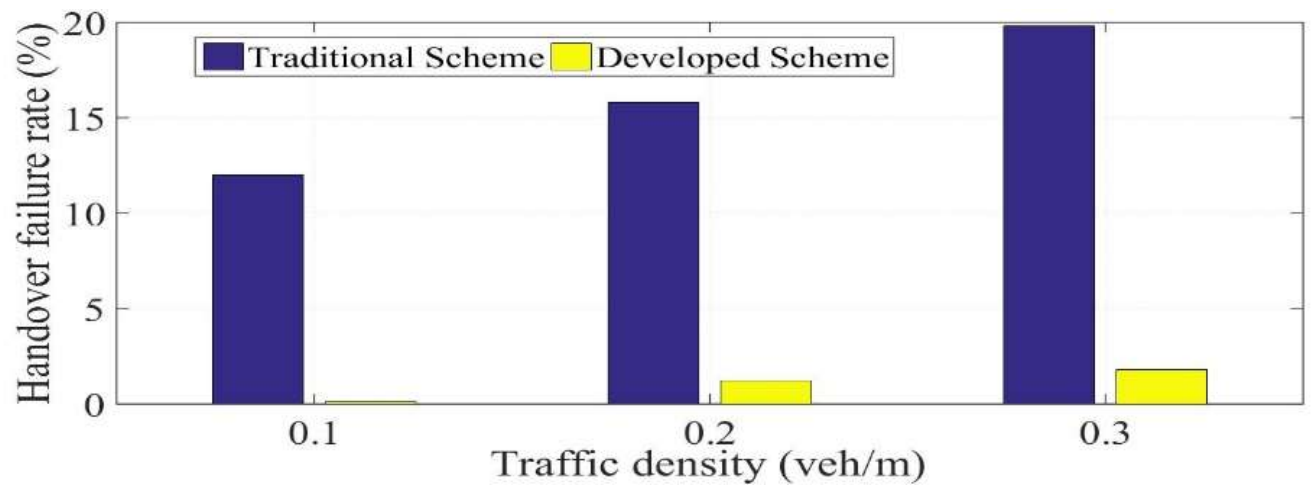

Figure 11. Handover failure rate of traditional handover schemes and the developed scheme at different traffic densities.

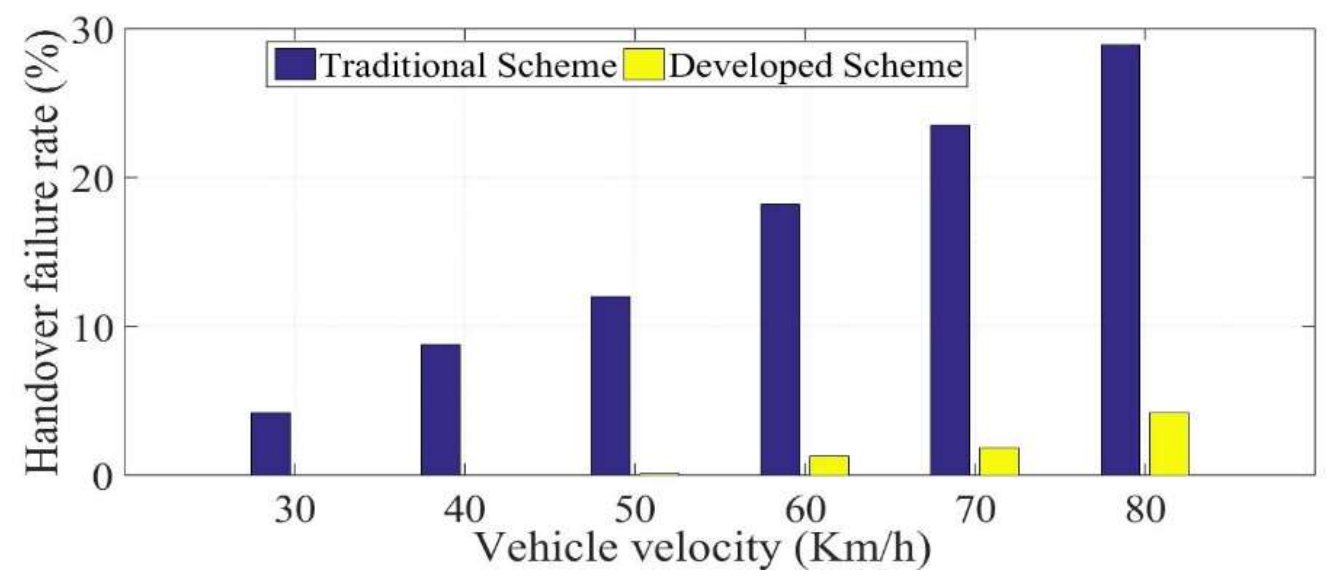

Figure 12. Handover failure rate of traditional handover schemes and the developed scheme at different vehicle velocities.

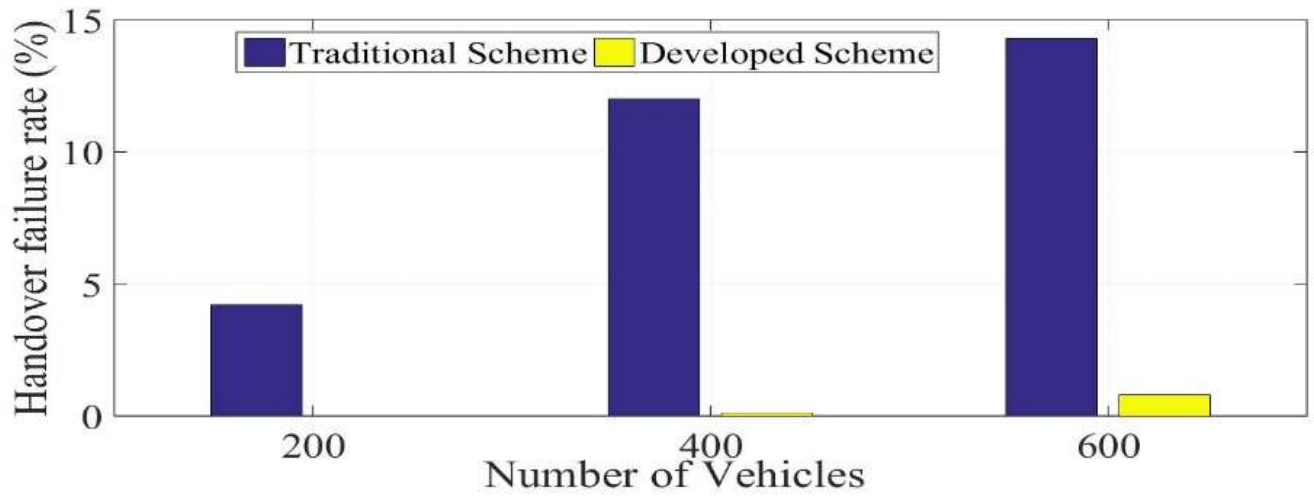

Figure 13. Handover failure rate of traditional handover schemes and the developed scheme with different numbers of deployed vehicles.

\section{Conclusions}

The handover process is a challenge in VANETs due to the high mobility conditions and the required user experience. This article introduced an MEC-based VANET structure 
and a seamless handover scheme for said structure. The handover process is implemented by an MEC server connected to an RSU, when vehicles come to the intersection of two coverage regions of neighboring RSUs. The developed scheme is based on three calculated threshold levels of received signal power in a way that ensures the handover process is performed before the received signal decreases below the reference threshold level that affects the packet reception. The developed scheme achieved a percentage improvement in the PDR of $64 \%$ compared to the existing traditional scheme. Moreover, the developed VANET achieved a $51 \%$ percentage improvement in blocked computing tasks compared to existing systems. The handover failure rate of the developed handover scheme was lower than the existing scheme by an average of $18 \%$. Our future directions for this work include analyzing and modifying the algorithm for crossroads with overlapped coverage results from multiple RSUs. This includes adding more metrics, such as the RSU load and the vehicle's predicted direction.

Author Contributions: Conceptualization, M.M.T., N.M. and A.A.A.; methodology, A.V. and F.E.A.E.-S.; software, M.M.T., A.M., N.M. and A.A.A.; validation, A.V., M.A.T., and A.A.A.; formal analysis, M.M.T. and N.M.; investigation, A.M. and A.V.; resources, F.E.A.E.-S.; data curation, M.A.T.; writing—original draft preparation, M.M.T., N.M. and A.A.A.; writing—review and editing, F.E.A.E.-S. and A.M.; visualization, A.M.; supervision, F.E.A.E.-S.; project administration, A.A.A.; funding acquisition, A.V. All authors have read and agreed to the published version of the manuscript.

Funding: Research funded by the Ministry of Digital Development, Communications, and Mass Media of the Russian Federation, contract number П33-1-26/9 (Moscow, Russia).

Institutional Review Board Statement: Not applicable.

Informed Consent Statement: Not applicable.

Data Availability Statement: The data presented in this study are available on request from the corresponding author.

Acknowledgments: The researcher A.A. Ateya is funded by the Ministry of Higher Education of the Arab Republic of Egypt.

Conflicts of Interest: The authors declare no conflict of interest. The funders had no role in the design of the study; in the collection, analyses, or interpretation of data; in the writing of the manuscript, or in the decision to publish the results.

\section{References}

1. Qiao, L.; Li, Y.; Chen, D.; Serikawa, S.; Guizani, M.; Lv, Z. A survey on 5G/6G, AI, and Robotics. Comput. Electr. Eng. 2021, 95, 107372. [CrossRef]

2. Dogra, A.; Jha, R.K.; Jain, S. A survey on beyond 5G network with the advent of 6G: Architecture and emerging technologies. IEEE Access 2020, 9, 67512-67547. [CrossRef]

3. Long, Q.; Chen, Y.; Zhang, H.; Lei, X. Software defined 5G and 6G networks: A survey. Mob. Netw. Appl. 2019, 1-21. [CrossRef]

4. Navarro-Ortiz, J.; Romero-Diaz, P.; Sendra, S.; Ameigeiras, P.; Ramos-Munoz, J.J.; Lopez-Soler, J.M. A survey on 5G usage scenarios and traffic models. IEEE Commun. Surv. Tutor. 2020, 22, 905-929. [CrossRef]

5. Siriwardhana, Y.; Porambage, P.; Liyanage, M.; Ylianttila, M. A Survey on Mobile Augmented Reality with 5G Mobile Edge Computing: Architectures, Applications, and Technical Aspects. IEEE Commun. Surv. Tutor. 2021, 23, 1160-1192. [CrossRef]

6. De Alwis, C.; Kalla, A.; Pham, Q.V.; Kumar, P.; Dev, K.; Hwang, W.J.; Liyanage, M. Survey on 6G frontiers: Trends, applications, requirements, technologies and future research. IEEE Open J. Commun. Soc. 2021, 2, 836-886. [CrossRef]

7. Mahmoud, H.H.H.; Amer, A.A.; Ismail, T. 6G: A comprehensive survey on technologies, applications, challenges, and research problems. Trans. Emerg. Telecommun. Technol. 2021, 32, e4233. [CrossRef]

8. Morgado, A.; Huq, K.M.S.; Mumtaz, S.; Rodriguez, J. A survey of 5G technologies: Regulatory, standardization and industrial perspectives. Digit. Commun. Netw. 2018, 4, 87-97. [CrossRef]

9. Jeong, J.; Shen, Y.; Oh, T.; Céspedes, S.; Benamar, N.; Wetterwald, M.; Härri, J. A comprehensive survey on vehicular networks for smart roads: A focus on IP-based approaches. Veh. Commun. 2021, 29, 100334. [CrossRef]

10. Ismael, H.R.; Ameen, S.Y.; Kak, S.F.; Yasin, H.M.; Ibrahim, I.M.; Ahmed, A.M.; Rashid, Z.N.; Omar, N.; Salih, A.A.; Ahmed, D.M. Reliable communications for vehicular networks. Asian J. Res. Comput. Sci. 2021, 10, 33-49. [CrossRef]

11. Zeadally, S.; Guerrero, J.; Contreras, J. A tutorial survey on vehicle-to-vehicle communications. Telecommun. Syst. 2020, 73, 469-489. [CrossRef] 
12. Al-Heety, O.S.; Zakaria, Z.; Ismail, M.; Shakir, M.M.; Alani, S.; Alsariera, H. A comprehensive survey: Benefits, services, recent works, challenges, security, and use cases for SDN-VANET. IEEE Access 2020, 8, 91028-91047. [CrossRef]

13. Arena, F.; Pau, G.; Severino, A. A review on IEEE 802.11 p for intelligent transportation systems. J. Sens. Actuator Netw. 2020, 9,22

14. Balador, A.; Cinque, E.; Pratesi, M.; Valentini, F.; Bai, C.; Gómez, A.A.; Mohammadi, M. Survey on decentralized congestion control methods for vehicular communication. Veh. Commun. 2021, 33, 100394. [CrossRef]

15. Ji, B.; Zhang, X.; Mumtaz, S.; Han, C.; Li, C.; Wen, H.; Wang, D. Survey on the internet of vehicles: Network architectures and applications. IEEE Commun. Stand. Mag. 2020, 4, 34-41. [CrossRef]

16. Bhatia, J.; Dave, R.; Bhayani, H.; Tanwar, S.; Nayyar, A. SDN-based real-time urban traffic analysis in VANET environment. Comput. Commun. 2020, 149, 162-175. [CrossRef]

17. Yu, H.; Liu, R.; Li, Z.; Ren, Y.; Jiang, H. An RSU Deployment Strategy based on Traffic Demand in Vehicular Ad Hoc Networks (VANETs). IEEE Internet Things J. 2021. [CrossRef]

18. Sharma, S.; Kaul, A. VANETs Cloud: Architecture, Applications, Challenges, and Issues. Arch. Comput. Methods Eng. 2021, 28, 2081-2102. [CrossRef]

19. Ahmed, A.A.; Alzahrani, A.A. A comprehensive survey on handover management for vehicular ad hoc network based on 5G mobile networks technology. Trans. Emerg. Telecommun. Technol. 2019, 30, e3546. [CrossRef]

20. Tayyab, M.; Gelabert, X.; Jäntti, R. A survey on handover management: From LTE to NR. IEEE Access 2019, 7, 118907-118930. [CrossRef]

21. Ahmad, R.; Sundararajan, E.A. A survey on femtocell handover management in dense heterogeneous 5G networks. Telecommun. Syst. 2020, 75, 481-507. [CrossRef]

22. Duo, R.; Wu, C.; Yoshinaga, T.; Zhang, J.; Ji, Y. SDN-based handover scheme in cellular/IEEE 802.11 p hybrid vehicular networks Sensors 2020, 20, 1082. [CrossRef] [PubMed]

23. Choi, J.H.; Han, Y.H.; Min, S.G. A network-based seamless handover scheme for VANETs. IEEE Access 2018, 6, 56311-56322. [CrossRef]

24. Pham, Q.V.; Fang, F.; Ha, V.N.; Piran, M.J.; Le, M.; Le, L.B.; Hwang, W.J.; Ding, Z. A survey of multi-access edge computing in 5G and beyond: Fundamentals, technology integration, and state-of-the-art. IEEE Access 2020, 8, 116974-117017. [CrossRef]

25. Filali, A.; Abouaomar, A.; Cherkaoui, S.; Kobbane, A.; Guizani, M. Multi-access edge computing: A survey. IEEE Access 2020, 8 , 197017-197046. [CrossRef]

26. Liu, L.; Chen, C.; Pei, Q.; Maharjan, S.; Zhang, Y. Vehicular edge computing and networking: A survey. Mob. Netw. Appl. 2020, 26, 1145-1168. [CrossRef]

27. Boukerche, A.; Soto, V. Computation offloading and retrieval for vehicular edge computing: Algorithms, models, and classification. ACM Comput. Surv. (CSUR) 2020, 53, 1-35. [CrossRef]

28. Cheng, X.; Huang, Z.; Chen, S. Vehicular communication channel measurement, modelling, and application for beyond 5G and 6G. IET Commun. 2020, 14, 3303-3311. [CrossRef]

29. Sharma, A.; Vanjani, P.; Paliwal, N.; Basnayaka, C.M.W.; Jayakody, D.N.K.; Wang, H.C.; Muthuchidambaranathan, P. Communication and networking technologies for UAVs: A survey. J. Netw. Comput. Appl. 2020, 168, 102739. [CrossRef]

30. Hussain, R.; Lee, J.; Zeadally, S. Trust in VANET: A survey of current solutions and future research opportunities. IEEE Trans. Intell. Transp. Syst. 2020, 22, 2553-2571. [CrossRef]

31. Hamdi, M.M.; Audah, L.; Rashid, S.A.; Mohammed, A.H.; Alani, S.; Mustafa, A.S. A review of applications, characteristics and challenges in vehicular ad hoc networks (VANETs). In Proceedings of the 2020 International Congress on Human-Computer Interaction, Optimization and Robotic Applications (HORA), Ankara, Turkey, 26-28 June 2020; IEEE: Manhattan, NY, USA, 2020; pp. 1-7.

32. Gyawali, S.; Xu, S.; Qian, Y.; Hu, R.Q. Challenges and solutions for cellular based v2x communications. IEEE Commun. Surv. Tutor. 2020, 23, 222-255. [CrossRef]

33. Jubara, H.E.I. An efficient handover procedure in vehicular communication. In Proceedings of the 20202 nd International Conference on Computer and Information Sciences (ICCIS), Sakaka, Saudi Arabia, 13-15 October 2020; IEEE: Manhattan, NY, USA, 2020; pp. 1-5.

34. Souza, V.B.; Pereira, M.H.; Lelis, L.H.; Masip-Bruin, X. Enhancing resource availability in vehicular fog computing through smart inter-domain handover. In Proceedings of the GLOBECOM 2020-2020 IEEE Global Communications Conference, Taipei, Taiwan, 7-11 December 2020; IEEE: Manhattan, NY, USA, 2020; pp. 1-6.

35. Chang, Y.H.; Liu, H.H.; Wei, H.Y. Group-based sidelink communication for seamless vehicular handover. IEEE Access 2019, 7, 56431-56442. [CrossRef]

36. Ge, X.; Li, Z.; Li, S. 5G software defined vehicular networks. IEEE Commun. Mag. 2017, 55, 87-93. [CrossRef]

37. Duo, R.; Wu, C.; Yoshinaga, T.; Ji, Y. SDN-based handover approach in IEEE $802.11 \mathrm{p}$ and LTE hybrid vehicular networks. In Proceedings of the 2018 IEEE SmartWorld, Ubiquitous Intelligence \& Computing, Advanced \& Trusted Computing, Scalable Computing \& Communications, Cloud \& Big Data Computing, Internet of People and Smart City Innovation (SmartWorld/SCALCOM/UIC/ATC/CBDCom/IOP/SCI), Guangzhou, China, 8-12 October 2018; IEEE: Manhattan, NY, USA, 2018; pp. $1870-1875$.

38. Muthanna, A.; Shamilova, R.; Ateya, A.A.; Paramonov, A.; Hammoudeh, M. A mobile edge computing/software-defined networking-enabled architecture for vehicular networks. Internet Technol. Lett. 2020, 3, e109. [CrossRef] 
39. Zhou, H.; Wang, H.; Chen, X.; Li, X.; Xu, S. Data offloading techniques through vehicular ad hoc networks: A survey. IEEE Access 2018, 6, 65250-65259. [CrossRef]

40. Baik, J.J.; Park, R.S.; Chun, H.Y.; Kim, J.J. A laboratory model of urban street-canyon flows. J. Appl. Meteorol. 2020, 39, 1592-1600. [CrossRef]

41. Vladyko, A.; Khakimov, A.; Muthanna, A.; Ateya, A.A.; Koucheryavy, A. Distributed edge computing to assist ultra-low-latency VANET applications. Future Internet 2019, 11, 128. [CrossRef] 